\title{
Bias effects in the possible/impossible object decision test with matching objects
}

\author{
Anja Soldan, H. John Hilton, and YaAkov Stern \\ Columbia University Medical Center, New York, New York
}

\begin{abstract}
In the possible/impossible object decision test, priming has consistently been found for structurally possible, but not impossible, objects, leading Schacter, Cooper, and Delaney (1990) to suggest that priming relies on a system that represents the global 3-D structure of objects. Using a modified design with matching objects to control for the influence of episodic memory, Ratcliff and McKoon (1995) and Williams and Tarr (1997) found negative priming for impossible objects (i.e., lower performance for old than for new items). Both teams argued that priming derives from (1) episodic memory for object features and (2) bias to respond "possible" to encoded objects or their possible parts. The present study applied the matched-objects design to the original Schacter and Cooper stimuli — same possible objects and matching impossible figures - with minimal procedural variation. The data from Experiment 1 only partially supported the bias models and suggested that priming was mediated by both local and global structural descriptions. Experiment 2 showed that negative priming for impossible objects derived from the structural properties of these objects, not from the influence of episodic memory on task performance. Supplemental materials for this study may be downloaded from mc.psychonomic-journals .org/content/supplemental.
\end{abstract}

Exposure to a stimulus or a related item can change how subjects respond to that stimulus on a subsequent exposure, even in the absence of conscious awareness of the previous encounter. This form of memory is referred to as priming and is thought to be distinct from explicit memory, or the ability to consciously recognize or recollect information (Schacter \& Buckner, 1998; Tulving \& Schacter, 1990). Priming is typically measured as a change in the speed, accuracy, or bias with which a stimulus is classified or identified on a repeated presentation.

Perceptual priming, in which the change in a subject's behavior is thought to result from a modification in the processing of the perceptual properties of a previously experienced stimulus, can be viewed not only as a form of memory, but also as a by-product of the normal operation of perceptual systems that learn (Bowers \& Marsolek, 2003; Henson, 2003). For this reason, the phenomenon of perceptual priming has informed the study of the nature of object representation and the different stages of perceptual analysis, particularly in the visual modality (for examples, see Bentin \& Golland, 2002; Biederman \& Cooper, 1991; Burgund \& Marsolek, 2000; Cooper \& Schacter, 1992; Gauthier \& Tarr, 1997; Itier \& Taylor, 2004).

A well-characterized perceptual priming paradigm that has been used to study how structural information about visual objects is represented is the possible/impossible object decision test developed by Cooper, Schacter, and colleagues (e.g., Schacter, Cooper, \& Delaney, 1990). The standard block-based implementation of this test consists of two phases: encoding and test. During the encoding phase, participants make a specific decision about unfamiliar line drawings; this decision is unrelated to the object decision performed at test. Half of the drawings depict structurally possible figures, which could exist as 3-D objects in the real world, and half represent structurally impossible figures, which cannot be rendered as coherent 3-D structures (see Figure 1). In the test phase, previously encoded figures are intermixed with an equivalent set of new possible and impossible items, and subjects are asked to classify them as either structurally possible or impossible. Priming has consistently been reported for possible objects in the form of greater classification accuracy or speed for old than for new test items. In contrast, impossible objects generally fail to elicit priming under standard test conditions (Liu \& Cooper, 2001; Schacter \& Cooper, 1993; Schacter et al., 1990; Schacter, Cooper, Delaney, Peterson, \& Tharan, 1991; Schacter, Cooper, Tharan, \& Rubens, 1991; Schacter, Cooper, \& Valdiserri, 1992; Soldan, Mangels, \& Cooper, 2008).

Although the basic behavioral finding of priming for possible, but not impossible, objects has been replicated many times, the underlying cause of this possible/ impossible difference has been the subject of much debate (e.g., Carrasco \& Seamon, 1996; Marsolek \& Burgund, 2005; Ratcliff \& McKoon, 1995; Schacter \& Cooper, 1995; Soldan, Mangels, \& Cooper, 2006; Williams \& Tarr, 1997). The original explanation by Schacter, Cooper, and colleagues proposed that the differential priming effects of possible and impossible objects arise from the operation of a structural description system (SDS), a 


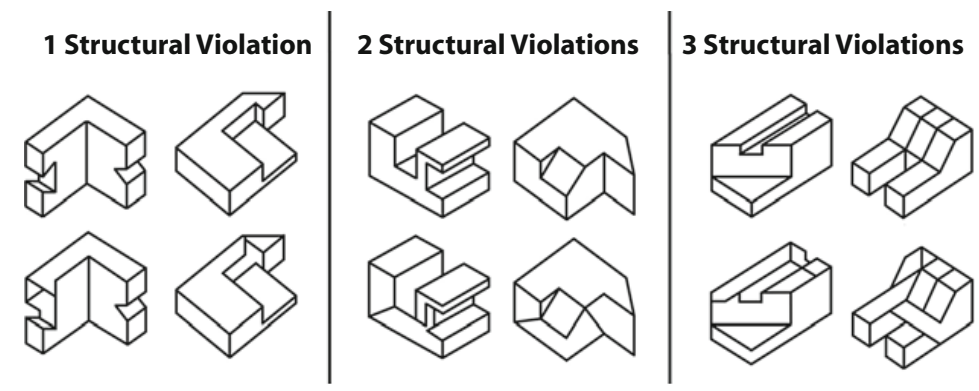

Figure 1. Examples of the matched possible and impossible objects used in this study. The upper row shows possible objects, and the lower row depicts the corresponding impossible objects with one (left panel), two (middle panel), or three (right panel) structural violations.

perceptual mechanism that can operate independently of explicit memory and is specialized for processing the 3-D structure of visual objects. Priming for possible objects is thought to result from facilitation in the processing of global structural descriptions of familiar, as compared with new, possible objects. Priming for impossible objects is not typically found under standard test conditions, because impossible objects lack a globally coherent 3-D geometry, precluding the SDS from creating a singular global 3-D representation. Thus, although possible parts of impossible objects are thought to be processed by and represented in the SDS, priming in the possible/impossible object decision test is thought to depend on access to information about the global organization of object parts (see, e.g., Schacter, Cooper, Delaney, et al., 1991).

Schacter and Cooper offered several lines of evidence to support their claim that priming in this test indeed reflects implicit visual perceptual mechanisms, as opposed to explicit memory or conceptual processes. First, performance in the possible/impossible object decision test is stochastically independent of old/new recognition performance (Schacter et al., 1990; Schacter, Cooper, Delaney, et al., 1991; but see Poldrack, 1996, for problems associated with testing for stochastic independence). Second, single and double dissociations between possible/ impossible object decision and old/new recognition have been observed by manipulating the encoding instructions (see, e.g., Schacter \& Cooper, 1993; Schacter et al., 1990) and by transforming object properties between encoding and test (Cooper, Schacter, Ballesteros, \& Moore, 1992; Schacter, Cooper, \& Treadwell, 1993). Third, priming for possible objects has been observed in amnesic patients with impaired explicit memory (Schacter, Cooper, Tharan, \& Rubens, 1991; Schacter et al., 1993).

Despite this evidence, two alternative accounts of possible/impossible object decision performance, in which retrieval from explicit memory plays an integral part, have been proposed (Ratcliff \& McKoon, 1995; Williams \& Tarr, 1997). Ratcliff and McKoon argued that Schacter and Cooper's findings with the possible/impossible object decision paradigm could be explained without recourse to an SDS. According to Ratcliff and McKoon's model, which we will refer to as the general bias model, the following contribute to possible/impossible object decision performance: (1) episodic memory for previously encoded object features and (2) a generic bias effect, defined as the increased probability of calling a test object "possible" when it is presented in the encoding phase, as compared with when it is not presented in the encoding phase. For possible objects, this bias increases classification accuracy for previously seen objects in comparison with new test objects (the positive priming effect), whereas, for impossible objects, it decreases classification accuracy for old objects in comparison with new objects (i.e., negative priming). However, negative priming is not typically found for impossible objects, because the bias effect is thought to be offset by episodic memory for specific object features that are associated with information about whether the object is possible or impossible. The nature of these features or configurations, however, was not specified by Ratcliff and McKoon and could consist of simple lines and angles, or more complex parts, boxes, or geons. We find it important that episodic memory for features and/or configurations is thought to improve classification accuracy for both item types. Thus, when the effects of bias and explicit memory are combined, there is no change in classification accuracy between old and new impossible objects. For old possible objects, bias and explicit memory positively combine to produce a robust enhancement in classification accuracy.

Williams and Tarr's (1997) structure-extraction bias model concurs with Ratcliff and McKoon (1995), in that possible/impossible object decision performance can be explained by a combination of explicit memory and bias. However, Williams and Tarr pointed out that the bias to respond "possible" to previously encoded objects is not the same for possible and impossible objects, but decreases systematically with the number of structural violations present in an object. They showed that possible objects presented at encoding produced more bias to respond "possible" at test than did impossible objects encoded with one structural violation, which in turn produced more bias than did impossible objects encoded with three structural violations. On the basis of these results, Williams and Tarr argued that bias is most appropriately considered to be an implicit memory phenomenon that results from a structureextraction system that is biased to process only the possible 
parts of an object. Unlike the SDS view, however, this system is thought to represent both possible and impossible objects in a strictly parts-based manner. It is not thought to represent global structural 3-D descriptions.

In support of their arguments, Ratcliff and McKoon (1995) and Williams and Tarr (1997) both utilized a paradigm with highly similar, matched possible and impossible objects to control for the influence of explicit memory on object decision performance. The encoding phase of this paradigm is identical to the standard possible/impossible object decision paradigm, but in the test phase, matched objects are included in addition to new and old possible and impossible objects. Matched objects are identical to objects presented in the encoding phase, except for one or a few features that alter the object's global 3-D structure from possible to impossible or vice versa. For the purpose of this article, matched possible object refers to a possible test object encoded in its impossible form; a matched impossible object is an impossible test object shown in its possible version at encoding. According to Ratcliff and McKoon, the high similarity between old and matched possible and impossible items makes recollection of episodic information at the time of test ineffective in helping to distinguish object possibility from object impossibility. Note, however, that this claim has never been tested.

When the influence of explicit memory was presumably reduced in this manner, Ratcliff and McKoon (1995) found that subjects exhibited only bias, consistent with their model. That is, subjects were more likely to label all encoded objects, whether possible or impossible, as possible, resulting in priming for possible objects (both old and matched) and negative priming for impossible objects (both old and matched). Williams and Tarr (1997) also showed an effect of bias for old and matched objects using this paradigm, but further demonstrated that the amount of bias decreased with the number of structural violations present in an object at encoding.

However, a recent study using event-related potentials to investigate the neural correlates of possible/impossible object decision performance failed to fully replicate the previously reported bias effects with the matched-objects design (Soldan et al., 2006). Although a bias to respond "possible" was found for old objects, no reliable bias was detected for the matched objects. This suggests that procedural differences between studies (e.g., stimulus durations, poststimulus masks, pacing and control of trials, balance of encoded vs. new stimuli) and differences in the particular stimulus sets may have significant effects on the experimental findings. Given that the matched-objects design was intended to address whether bias could indeed account for the original Schacter and Cooper findings, it should be applied to the original Schacter and Cooper design and stimuli.

Therefore, the primary aim of the present study was to replicate the matched-objects design in a large sample of subjects using the same experimental procedures and objects that Schacter and Cooper (1993) used (i.e., the same possible objects and matching impossible objects). If bias does indeed account for the original Schacter and Cooper results, all old and matched objects, whether possible or impossible, should evoke possible responses consistent with the Ratcliff and McKoon (1995) and Williams and Tarr (1997) findings. As in the original studies by Schacter and Cooper (e.g., Schacter, Cooper, Delaney, et al., 1991), in the present study, there was high interrater agreement about the possible/impossible status of the objects (see the Results section). This is of critical importance because, unless the global 3-D structure of a possible object (or lack thereof in an impossible object) can clearly be identified, one cannot make conclusions regarding the effects of global object structure on perceptual priming. Note that in the study by Ratcliff and McKoon, the possible/impossible status of the objects was relatively ambiguous (the mean classification accuracy, given unlimited time, was only $78 \%$ for possible and $76 \%$ for impossible objects), which may have limited the generality of their findings.

Second, we introduced a delay manipulation to further evaluate the effect of explicit memory on possible/ impossible object decision performance. Previous work has consistently shown that increasing the delay between the time that stimuli are encoded and the time when memory is tested decreases performance on explicit tests of memory (e.g., Wixted \& Ebbesen, 1991), with the greatest decreases occurring in the first 15 to $20 \mathrm{~min}$ (e.g., McBride \& Dosher, 1997; see also Stern et al., 2000). Thus, unless the influence of explicit memory is completely eliminated by the matched-objects manipulation, the bias view would predict an increase in the bias to respond "possible" as the encoding-to-test delay increases, because of the concomitant decrease in explicit memory.

Third, we compared priming for old possible and impossible objects in the presence (Experiment 1) and absence (Experiment 2) of the matched objects in order to confirm that priming for old objects would, in fact, change with the introduction of the matched objects. Such a change would be predicted according to the bias models if the matched objects indeed influence the degree to which subjects rely on explicit memory to perform the possible/impossible object decision test. Note that the same experimental design implemented in the present study, including the delay manipulation, has been used in an experiment with the original nonmatching possible and impossible objects developed by Schacter and Cooper and has been shown to produce priming for possible, but not impossible, objects across all tested encoding-to-test delays in young adults (Soldan, Hilton, Cooper, \& Stern, in press). ${ }^{1}$ This means that any difference in the pattern of priming between the present results and those obtained in the Soldan et al. (in press) study can only be attributed to the matched-objects manipulation.

\section{EXPERIMENT 1}

\section{Method}

Subjects. Ninety-six young volunteers (66 female), who were from 18 to 30 years of age $(M=19.9, S D=2.0)$, participated in this experiment. Subjects were drawn from the student population at Columbia University and received either $\$ 10 / \mathrm{h}$ or partial course credit for Introduction to Psychology. The mean number of years of education was $13.6(S D=1.3)$. Half of the subjects $(n=48)$ were assigned to the 20-min delay condition, the other half to the 90-min delay condition. Three subjects were replaced because of run-time 
errors, and an additional 4 were replaced because their performance in the possible/impossible object decision test did not differ from chance $(p>.3)$. All subjects were fully debriefed upon completion or termination of the experiment.

Stimuli. Ninety-six pairs of line-drawn novel objects were used as experimental stimuli. All possible objects were directly adopted from the original set of 124 possible objects from which Schacter and Cooper (Cooper, 1990; Schacter, Cooper, Delaney, et al., 1991; Penney, Mecklinger, Hilton, \& Cooper, 2000) selected their stimuli. However, because Schacter and Cooper did not have a set of matching possible and impossible objects, matched impossible versions were created by adding one or more structural violations to an original possible object. The reverse approach — keeping the original Schacter and Cooper impossible objects and creating matching possible objects - was not feasible because, for many impossible objects, it was extremely difficult to generate a globally coherent, yet highly similar, 3-D object, particularly for those with three or more structural violations. In addition, the structural violations in some of the impossible figures in the Schacter and Cooper stimulus set were such that they made the figure appear flat, or 2-D, and were therefore too dissimilar to a possible version of that figure.

The possible and impossible versions of an object were matched with respect to the number of visible surfaces, lines (i.e., straight lines needed to construct the figure \pm 1 ), line segments (i.e., the distance from one intersection to another \pm 1 ), and vertices (i.e., intersections of two or more line segments \pm 1 ). Furthermore, the possible and impossible versions shared identical bounding contours. The impossible objects consisted of 32 objects with one structural violation, 30 with two structural violations, and 34 with three structural violations. The discriminability of the possible and impossible objects was high, as determined in a preliminary study with 24 other subjects: Subjects classified $95 \%$ of the possible objects as possible (given unlimited time) and $91 \%$ of the impossible objects as impossible (only 3 possible and 4 impossible objects scored below $70 \%$ correct across subjects). An additional 6 items ( 3 possible and 3 impossible) were used as buffer items at the beginning of the encoding and test blocks. In a second baseline study, 10 additional subjects viewed the 96 pairs of objects at the brief duration $(33 \mathrm{msec}$ ) used in the test phase of the experiment and classified them as either possible or impossible. On the basis of these results, objects were assigned to eight different object sets, so that differences in mean accuracy and $S D$ across object sets were minimized. The assignment of object sets to encoding conditions was counterbalanced across subjects, so that each object occurred equally often in each of the encoding conditions (old immediate, old delayed, matched immediate, matched delayed, new).

Note that as a result of creating new matching impossible objects that were highly similar to the original possible objects, the overall composition of the impossible objects used in the present study was somewhat different from that of the original impossible objects in the Schacter and Cooper stimulus set. In particular, the proportions of impossible objects with one, two, or three structural violations were $33 \%, 31 \%$, and $35 \%$, respectively. By comparison, the set of impossible objects $(n=152)$ from which Schacter and Cooper chose their stimuli consisted of approximately $13 \%, 28 \%$, and $59 \%$ of stimuli with one, two, or three or more structural violations, respectively. Likewise, in Soldan, Hilton, Cooper, and Stern's (in press) Experiment 1, which used the original Schacter and Cooper stimuli with the same design as in the present study, the proportions of impossible objects $(n=48)$ with one, two, or three or more structural violations were $13 \%, 29 \%$, and $58 \%$, respectively. This change in the composition of the impossible stimuli was unavoidable because we desired a similar proportion of objects with one, two, or three structural violations in order to test the predictions of the structure-extraction bias model.

Procedure. The experiment consisted of two encoding blocks and one subsequent test block. The first encoding block was presented either 20 or 90 min prior to test, and the second occurred immediately prior to test. Distinct sets of 24 objects (12 possible, 12 impossible) were shown at the two encoding sessions. Thus, all subjects encoded a set of objects immediately prior to test: Half of them encoded an additional set $20 \mathrm{~min}$ prior to test, and the remaining half encoded the additional set $90 \mathrm{~min}$ before test. The task at encoding was to indicate whether the presented object faced primarily toward the left or toward the right. Although there is no objectively right or wrong answer in this task, it is thought to require that subjects process the global structure of the object, and it has been shown to produce robust priming for possible objects under standard test conditions (Cooper \& Schacter, 1992; Schacter \& Cooper, 1993). Ratcliff and McKoon (1995) and Williams and Tarr (1997) also used this task in their matched objects studies. During the encoding phases, no mention was made of the distinction between possible and impossible objects or of the subsequent object decision test. The encoding trials were self-initiated by a press of the space bar and began with a fixation cross ("+") shown at the center of the screen for $350 \mathrm{msec}$. A single stimulus was then presented for 4,000 msec, and the subjects were directed to enter their left/right decision following stimulus offset, using the "z" or "/" key with their left or right hand, respectively.

At test, the subjects viewed all 48 encoded objects (12 possible and 12 impossible from the first encoding session, 12 possible and 12 impossible from the second encoding session), the matched mates of the 48 encoded objects, and 96 new objects ( 48 objects, in both possible and impossible versions) in an intermixed sequence. The task was to indicate as quickly and as accurately as possible, using the " $\mathrm{z}$ " or "/" key with their left or right hand, respectively, whether an item represented a structurally possible or impossible object. Trials were again self-initiated by a space bar press and began with a $150-\mathrm{msec}$ fixation cross. Each test stimulus was then displayed for $33 \mathrm{msec}$ (unmasked, 2 refresh cycles on the computer screen). With this exposure duration, baseline classification accuracy for new items was approximately between $60 \%$ and $80 \%$, which left sufficient room to observe priming (both positive and negative) and ensured that subjects could perform the task reliably better than chance. Note that in order to obtain similar baseline rates in performance, Ratcliff and McKoon (1995) used longer test exposures (i.e., 150-250 msec, unmasked), Williams and Tarr (1997) used shorter ones (i.e., $45 \mathrm{msec}$, backward masked), and prior studies by Schacter and Cooper used exposure durations in the range of 17 to $100 \mathrm{msec}$ (see, e.g., Schacter \& Cooper, 1993). This variability in stimulus exposure durations likely reflected differences in stimulus sets, participant populations, and display monitors (e.g., refresh rate, resolution).

As in Williams and Tarr (1997), this study presented both the possible and the impossible versions of an object during the test phase in order to increase the total number of trials per subject. On the basis of the results by Williams and Tarr, we expected the first presentation of an object at test to have no significant impact on the second presentation of that object in its other version. Ratcliff and McKoon (1995) showed only one member of a pair at test.

The experiment was run on a Macintosh PowerPC 650 computer using the PsyScope 1.2.5 experiment development package. The screen resolution was set to $640 \times 480$, and the objects were drawn so that they fit completely within a circle that subtended $11^{\circ}$ of visual angle when viewed at a distance of approximately $60 \mathrm{~cm}$.

Data analysis. Consistent with the studies by Schacter, Cooper, and colleagues (e.g., Schacter \& Cooper, 1993), Williams and Tarr (1997), and Ratcliff and McKoon (1995), accuracy served as the primary dependent variable in our analysis. A preliminary analysis indicated that there were no significant effects for reaction time, so reaction time is not discussed further. In addition, to facilitate comparison of our results with those by Williams and Tarr, we also analyzed the accuracy data in terms of the signal detection measures of bias $\left(C_{\mathrm{L}}\right)$ and sensitivity $\left(d_{\mathrm{L}}\right)$ (Snodgrass \& Corwin, 1988). Sensitivity corresponds to the ability to discriminate between the distributions of possible and impossible items, whereas bias refers to the degree to which subjects use one type of response (i.e., "possible") more than another response (i.e., "impossible"). In particular, more positive $d_{\mathrm{L}}$ scores indicate greater sensitivity, and more negative $C_{\mathrm{L}}$ scores indicate a greater bias to respond "possible."

It is also worth mentioning that even though the signal detection theory measures can be useful in interpreting data of this type- 
particularly with respect to the bias models of possible/impossible object decision priming - the interpretation of these measures is strongly dependent on the assumptions (often untested) one makes regarding the underlying dimension(s) along which subjects are assumed to classify possible and impossible objects. For example, Williams and Tarr (1997) assumed that subjects classified possible and impossible objects on the basis of the "amount of structural evidence" in test objects, whereas Marsolek and Burgund (2005) assumed that classifications were made according to a "strength of feeling of possibility." In the present study, we do not favor one assumption over another; we merely report these measures as a means for comparison with prior and future results. All reported $p$ values for post hoc and planned comparisons were corrected for multiple comparisons using the Bonferroni-Holm correction.

\section{Results}

Accuracy analysis. For both the immediate and delayed encoding conditions, accuracy priming scores for old and matched possible and impossible objects were computed for each subject by subtracting the percentage of correct new trials from the percentage of correct old trials (for old objects) or from the percentage of correct matched trials (for matched objects). A preliminary analysis showed the presence of an interaction between test order, encoding status, and delay condition $[F(1,94)=$ $4.41, p=.039]$, indicating that the initial presentation of one member of an object pair may have influenced subjects' responses to the subsequent presentation of the other member of that object pair. Therefore, all analyses and means reported here are limited to the first presentation of a member of an object pair. Except for this one interaction involving test order, however, the pattern of results was the same whether the analysis included both members of an object pair or only the first presentation.

Mean object-classification accuracy for possible and impossible objects (as a function of encoding status and encoding-to-test delay) are shown in Figure 2 for the 20min and 90-min delay length groups. A repeated measures ANOVA with delay length group (20 vs. $90 \mathrm{~min})$, object type (possible vs. impossible), encoding status (old vs. matched), and delay condition (immediate vs. delayed) as factors indicated more positive priming scores for possible than for impossible objects $[F(1,94)=8.58, p<$ $.005]$. There was also a main effect of encoding status $[F(1,94)=5.82, p=.018]$, indicating more positive priming scores for old than for matched objects, and an interaction between encoding status and object type $[F(1,94)=$ $3.83, p=.05]$. None of the effects involving delay and delay group were significant (all $p \mathrm{~s}>.12$ ). Therefore, all subsequent analyses were collapsed across these two variables in order to increase statistical power.

Post hoc $t$ tests revealed that the priming scores for old possible objects were more positive than the priming scores for the three other object types (all $p \mathrm{~s}<.0005$ ). However, there was no difference in the magnitude of priming between matched possible, old impossible, and matched impossible objects (all $p s>.2$ ). Additional planned comparisons (collapsing across the delay variables) indicated highly significant positive priming for old possible objects $[M=5.41, S E=1.13 ; t(95)=4.87, p<.0001]$, but not for matched possible objects $[M=-0.73, S E=1.51 ; t(95)=$

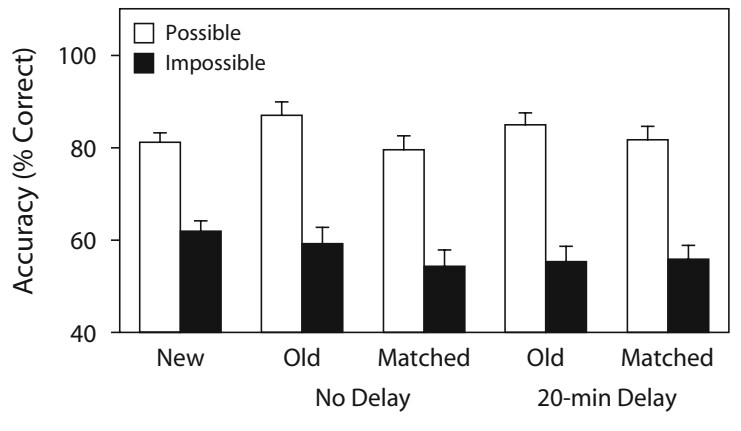

Encoding Status

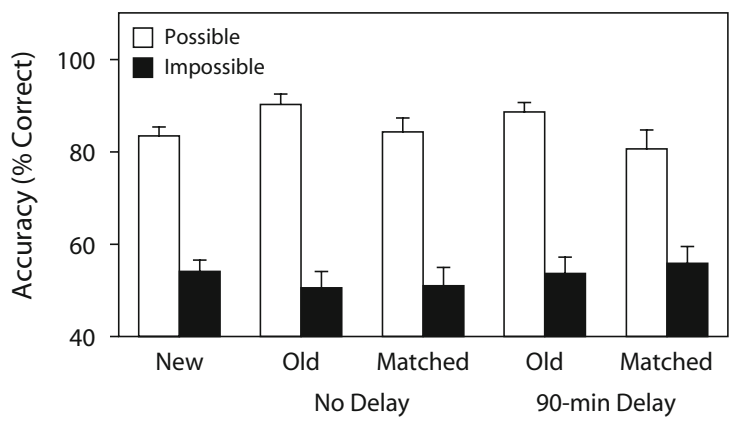

Encoding Status

Figure 2. Results from Experiment 1: Mean object decision accuracy, as a function of encoding status for subjects in the 20-min (upper panel) and 90-min (lower panel) delay groups. Error bars represent the standard errors of the means.

$0.48, p=.63]$. For impossible test objects, negative priming approached, but did not reach, significance, whether the objects were old $[M=-3.35, S E=2.02 ; t(95)=1.66$, $p=.10$, uncorrected $]$ or matched $[M=-3.76, S E=1.96$; $t(95)=1.92, p=.06$, uncorrected]. However, when floor effects were taken into account by eliminating subjects with classification accuracies of less than $40 \%$ for new impossible objects $(n=15)$, the magnitude of negative priming for old impossible $[M=-5.22, S E=2.22 ; t(80)=2.36$, $p<.05]$ and matched impossible $[M=-5.26, S E=2.15$; $t(80)=2.45, p<.05$ ] objects was significant. There was no difference in priming between the two types of impossible objects $(p>.9)$.

As shown in Figure 3 and predicted by the structureextraction bias view, negative priming for impossible objects was inversely related to the number of structural violations in the objects - particularly for old impossible objects. This was confirmed by a repeated measures ANOVA on the priming scores of impossible objects, with two levels of encoding status (old vs. matched) and three levels of structural violations (1 vs. 2 vs. 3 ). The existence of a negative linear relation between number of structural violations and magnitude of negative priming was assessed with a first-order polynomial contrast. Although there were no significant effects of encoding status and number of structural violations (all $p \mathrm{~s}>.20$ ), the firstorder polynomial contrast was significant $[F(1,95)=$ 


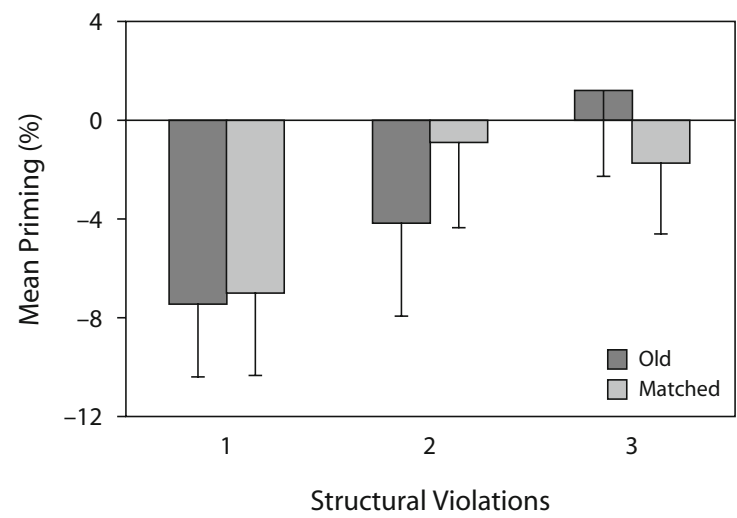

Figure 3. Results from Experiment 1: Mean priming scores for old and matched impossible objects, as a function of the number of structural violations in the object. Error bars represent the standard errors of the means.

$3.24, p=.038$, one-tailed]. Planned comparison $t$ tests averaging over old and matched items showed that negative priming was significant for impossible objects with one structural violation $[M=-7.54, S E=2.59 ; t(95)=$ $2.91, p<.01$ ], but not for impossible objects with two or three structural violations (both $p \mathrm{~s}>.3$ ).

Because the bias models predicted priming for matched possible objects, we examined whether the lack of priming for these stimuli in this experiment was related to ceiling effects for new objects, which was at $95 \%$ or higher for several subjects. When subjects with classification accuracies of $95 \%$ or higher for new possible items $(n=22)$ were eliminated, priming for matched possible objects was still not significant $[M=0.22, S E=1.91 ; t(73)=0.11, p=$ .91], whereas priming for old possible objects was highly significant $[M=7.26, S E=1.35 ; t(73)=5.38, p<.0001]$. Because the structure-extraction bias view holds that priming should be maximal for objects with fewer structural violations, we also tested whether priming would occur for matched possible objects that were encoded with a single structural violation. This analysis again showed no evidence of priming; in fact, it showed a trend in the opposite direction $[M=-3.68, S E=2.86 ; t(95)=1.29, p>.2]$.

Signal detection analysis. The subjects' mean sensitivity and bias scores for new, old, and matched objects are shown in the top panel of Figure 4. For consistency with the accuracy data, we computed priming scores for the bias $\left(C_{\mathrm{L}}\right)$ and sensitivity $\left(d_{\mathrm{L}}\right)$ measures that represented the difference in performance (i.e., bias or sensitivity) for new items and old items or matched items. These priming scores were then submitted to a repeated measures ANOVA with delay-length group, encoding status, and delay condition as variables. For sensitivity, the only significant effect was that of encoding status $[F(1,94)=7.05$, $p=.009]$; sensitivity was more positive for old objects than for matched objects. According to post hoc $t$ tests, the increase in sensitivity for old relative to new objects was significant when ceiling and floor effects were taken into account by removing all subjects $(n=24)$ with classifica- tion accuracy greater than or equal to $95 \%$ or less than or equal to $25 \%$ for new possible or impossible objects $[t(71)=2.67, p=.018$; without adjustment for floor and ceiling effects, $p=.2]$. Sensitivity for matched objects did not differ from zero, even when floor and ceiling effects were eliminated $(p>.2)$.

For bias, there was also a main effect of encoding status $[F(1,94)=4.41, p=.039]$, but no other significant effects (all $p \mathrm{~s}>.15$ ), reflecting more negative bias scores (i.e., a greater bias to respond "possible") for old objects than for matched objects. According to a post hoc $t$ test, the bias to respond "possible" to old objects was significantly greater than that to new objects, indicating the presence of priming $[t(95)=3.65, p=.0008]$. However, there was no difference in bias for new objects and matched objects $[t(95)=0.87, p=.39]$, even when floor and ceiling effects were taken into account.

Next, we computed separate priming scores for old objects for which the impossible version of the object had one, two,
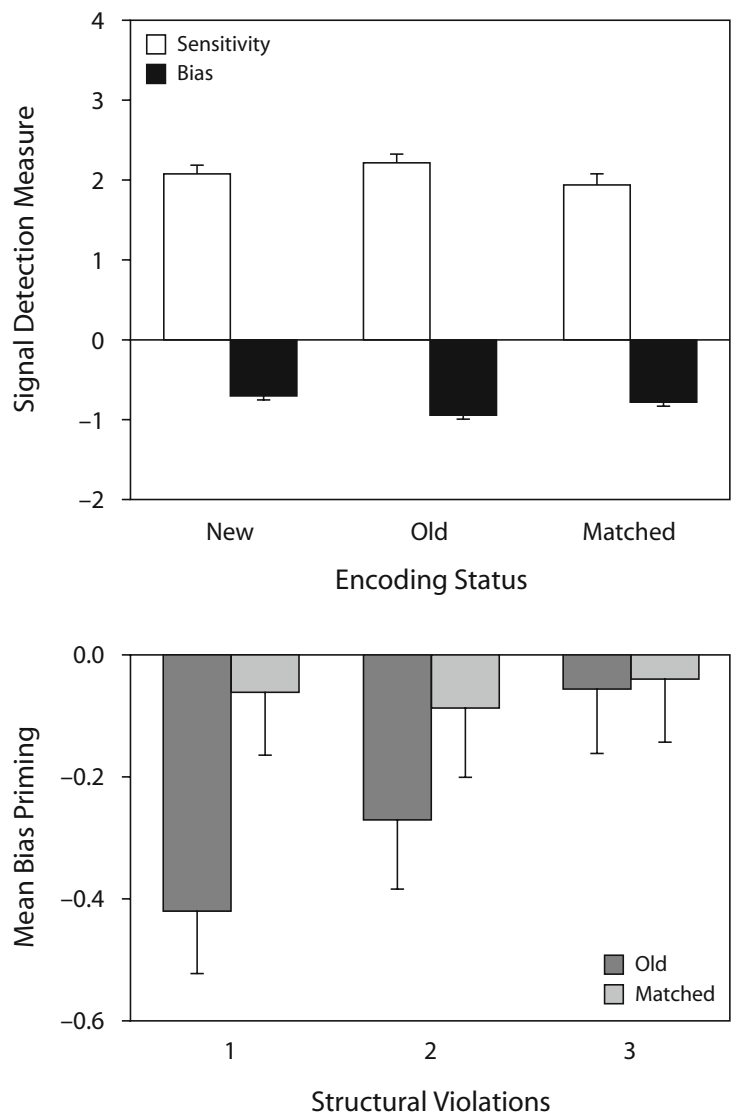

Figure 4. Results from Experiment 1: Mean bias and sensitivity scores for new, old, and matched objects (top panel). The difference in bias between new and old objects (i.e., bias priming score) decreased linearly as the number of structural violations of impossible objects increased (bottom panel). For the matched objects, bias priming was unaffected by the number of structural violations and did not differ from zero (bottom panel). Error bars represent the standard errors of the means. 
or three structural violations. Consistent with the structureextraction bias view, the effect of number of structural violations (1 vs. 2 vs. 3 ) on bias approached significance $[F(1,94)=2.94, p=.056]$, and the first-order polynomial contrast testing for a significant linear increase in bias with decreasing number of structural violations was significant $[F(1,94)=5.97, p=.016]$. For sensitivity, the linear contrast did not approach significance, even when ceiling and floor effects were taken into account (all $p \mathrm{~s}>.3$ ). When the same analyses were conducted for the matched objects, no significant effects were detected for sensitivity and bias (all $p \mathrm{~s}>.3$ ). Mean bias priming scores for old and matched objects (as a function of the number of structural violations) can be found in the bottom panel of Figure 4.

\section{Discussion}

In summary, we found significant positive priming for old possible objects and significant negative priming for old impossible and matched impossible objects when floor effects were taken into account. However, there was no evidence for priming of matched possible objects. This indicates that when presented with a possible test object, subjects were more likely to classify it as possible only if they had viewed the possible object during encoding, but not if they had viewed an impossible version of that possible object. By contrast, when shown an impossible test object, subjects were more likely to classify the object as possible when they saw the same impossible object or a possible version of that object at encoding. Moreover, the magnitude of negative priming of impossible objects decreased as the number of structural violations in the object increased. The signal detection measures indicated that subjects' sensitivity in discriminating between possible and impossible objects was greater for old than for new objects when ceiling effects for new items were taken into account. Likewise, subjects' tendency to respond "possible" was greater for old than for new test items. This increase in bias to respond "possible" to old as opposed to new objects was negatively related to the number of structural violations in the objects, so that the greatest bias occurred for objects with one structural violation and the least bias occurred for objects with three structural violations. There was no difference in bias or sensitivity between new objects and matched objects. Furthermore, there was no evidence for an effect of delay on task performance.

Although several aspects of these results are in accord with the bias models, other findings are inconsistent with them. As predicted by the bias models, priming was observed for old possible objects and negative priming for old impossible objects, indicating that subjects were biased to respond "possible" to all old objects (both possible and impossible). This was confirmed by an increase in bias to respond "possible" to old as opposed to new objects, as measured by $C_{\mathrm{L}}$. Furthermore, the magnitude of priming for old objects, as measured by bias $\left(C_{\mathrm{L}}\right)$, decreased with an increase in the number of structural violations in the objects, which is also consistent with the structure-extraction bias account. Additional support for the bias models is the finding that negative priming occurred for matched impossible objects when floor ef- fects were removed - particularly those objects with one structural violation. Also consistent with the structureextraction bias model is the observed increase in sensitivity to old relative to new objects. The reason, according to this model, is that possible objects contain more valid structural information that can be primed than do impossible objects. This leads to more positive priming of old possible objects than negative priming for old impossible objects, which results in a net increase in sensitivity to old items relative to that to new items.

However, the lack of priming for matched possible objects is not consistent with the notion of a simple increase in bias to respond possible to all objects that contain familiar parts, as hypothesized by the bias models. Even when an object contained a single structural violation during encoding, subjects were not more likely to classify the object as possible when presented the possible version of the object at test. Note also that the absence of a priming effect for matched possible objects is unlikely to be due to insufficient similarity or structural overlap between the possible and impossible members of an object pair. If this were the case, priming should also not have occurred for matched impossible objects. Moreover, a power calculation indicated that the observed absence of priming for matched possible objects was also unlikely to be due to insufficient power to detect such an effect. The power to detect a priming effect for matched possible objects just two thirds the size of that observed for old possible objects was large (power $=0.8, N=96, p<.05$, one-tailed). This suggests that, contrary to the bias models, there might not be just a quantitative difference in the way possible and impossible objects are encoded (i.e., on the basis of the amount of valid parts-based structural information), but also a qualitative difference (i.e., on the basis of the presence or absence of global 3-D structural descriptions) as well, as suggested by the SDS model.

Another aspect of the present results that does not support the bias models is the finding that the bias to respond "possible" was not higher for matched objects than for new objects. Thus, although we observed negative priming of matched impossible objects when floor effects were eliminated, there was a nonsignificant increase in subjects' bias to respond "impossible" to matched possible objects, resulting in no net change in the bias to respond "possible" to matched items. Both bias models would have predicted an increase in the bias to respond "possible" to all familiar items, old and matched.

Although the results from Experiment 1 are inconsistent with the bias models in several respects, they are not fully consistent with the SDS model either. According to the SDS model, priming in the possible/impossible object decision test reflects facilitated processing of the global 3-D structure of objects. The results for possible test objects are therefore consistent with the SDS model, which would predict priming for old possible, but not matched possible, test objects, because only in the case of old possible objects were subjects able to encode and later access the global structural descriptions of the objects. In the case of matched possible objects presented in their impossible version at encoding, such global structural in- 
formation cannot be encoded, and, therefore, no priming would be expected. The finding of negative priming for old and matched impossible objects, however, is not consistent with the SDS model, because these stimuli do not contain a globally coherent structure that can be primed.

In defense of the SDS model, one could argue that the negative priming effect for matched impossible objectsparticularly those with a single structural violation-was mediated by the global structure of the matching possible object that the subject viewed at encoding, rather than by local parts. This could occur if a subject missed the single structural violation in a briefly presented matched impossible object and perceived the previously encoded global structure instead. This scenario, however, would imply that two different processes give rise to priming for matched and old impossible objects: one based on the global structure of the possible counterpart (for matched impossible objects) and one based on local structural information (for old impossible objects). This, however, is a less parsimonious explanation than the notion that responses to all impossible test objects are based on local structural information.

The finding of negative priming for old impossible objects further suggests that the inclusion of the matched objects in the test phase may have influenced object decision performance for old items. Thus, when the original nonmatching possible and impossible stimuli developed by Schacter, Cooper, and colleagues were used with the same experimental design as that used in Experiment 1 of Soldan et al. (in press), we found the typical pattern of results: priming for possible objects and nil priming for impossible objects. Given that the only procedural difference between these two experiments was the addition of the matched objects during the test phase in Experiment 1 of the present study, the negative priming effect for old impossible objects in our Experiment 1 could be attributable to the fact that the matched objects reduced the putative influence of explicit memory on task performance, as postulated by Ratcliff and McKoon (1995) and Williams and Tarr (1997). The reduction in explicit memory may, in turn, have exposed the bias to respond "possible" to all old items, leading to priming for old possible objects and negative priming of old impossible objects.

Another possibility is that the negative priming effect for old impossible objects in Experiment 1 was due to stimulus-specific characteristics. Thus, although all of the possible objects used in this study were directly adopted from the original stimulus set of Schacter, Cooper, and colleagues, new impossible objects had to be constructed in order to create matching possible and impossible pairs (see the Method section for our Experiment 1). The impossible objects used in this study, however, had fewer structural violations than did the impossible objects used in the study with nonmatching possible and impossible figures (see the Method section for our Experiment 1). Given that negative priming appears to be inversely related to the number of structural violations of an impossible object, one would expect to find more negative priming for the impossible objects used in this study than for the original impossible objects in the Schacter and Cooper stimulus set. Therefore, the significant negative priming effect for old impossible objects in Experiment 1 may simply reflect the greater proportion of impossible objects with one or two structural violations, rather than the influence of the matched objects on object decision performance for old items. The goal of Experiment 2 was to adjudicate between these two hypotheses.

\section{EXPERIMENT 2}

According to the bias models, removal of the matched objects from the test phase should increase the magnitude of priming for old possible objects because bias and explicit memory positively combine and should decrease the magnitude of negative priming for old impossible objects because bias and explicit memory counteract each other. In signal detection terminology, the bias to respond "possible" should be smaller when no matching objects are present than when matching objects are present.

Ratcliff and McKoon (1995) previously compared possible/impossible object decision priming in the presence and in the absence of the matched objects and found negative priming for old and matched impossible objects only when the matched objects were present, strongly supporting the bias models. However, in light of the low discriminability of their possible and impossible objectseven under unlimited viewing conditions (classification accuracy was only $78 \%$ for possible and $76 \%$ for impossible objects) - their results may not generalize to conditions where possible and impossible objects are clearly differentiable. By comparison, Williams and Tarr (1997) used well-differentiable possible and impossible objects but did not compare object decision performance in the presence and the absence of matched objects. Thus, it remains to be seen whether matched objects have an effect on possible/ impossible object decision performance when the possible and impossible objects are clearly differentiable.

\section{Method}

Subjects. Twenty-four subjects participated in Experiment 2. All subjects were between 18 and 30 years of age, right-handed, and healthy, and all reported having normal or corrected-to-normal vision.

Procedure. The encoding phase was identical to that of Experiment 1 , except that all 48 objects ( 24 possible, 24 impossible) were shown in the same encoding session, which occurred immediately before the test phase. The test phase was also the same as that in Experiment 1 , except that only old $(n=48)$ and new $(n=48)$, but no matched, objects were presented. In other words, this is the standard object decision paradigm. There were four different groups of 24 possible and 24 nonmatching impossible objects that were rotated across participants, so that each object appeared equally often in each condition.

\section{Results}

As can be seen in Figure 5, mean object decision accuracy for possible and impossible objects, as a function of encoding status (new vs. old), was very similar to that found in Experiment 1. The results were analyzed using repeated measures ANOVAs with object type (possible vs. impossible) and encoding status (new vs. old) as withinsubjects factors. This analysis revealed that classification accuracy was higher for possible than for impossible 


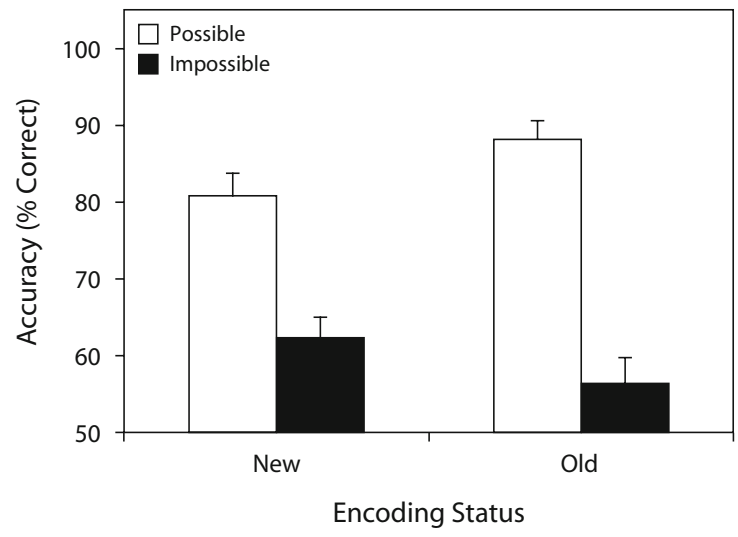

Figure 5. Results from Experiment 2: Mean object decision accuracy, as a function of encoding status. Error bars represent the standard errors of the means.

objects $[F(23)=25.81, p<.0001]$ and that there was an interaction between object type and encoding status $[F(23)=15.04, p<.001]$. Planned comparison $t$ tests showed that priming occurred for possible objects $[M=$ $7.30, S E=2.12 ; t(23)=3.45, p=.002]$ and that negative priming occurred for impossible objects $[M=-6.08$, $S E=2.59 ; t(23)=2.34, p=.03]$. A comparison across Experiments 1 and 2 showed that there was no main effect of experiment on object decision accuracy for new and old objects, nor any interaction involving experiment (all $p \mathrm{~s}>.3$ ). A direct comparison of the priming scores for old possible and old impossible objects across experiments also indicated no reliable differences (both $p$ s $>$.4).

Next, we analyzed whether negative priming for impossible objects was dependent on the number of structural violations in the objects. Separate priming scores (old new) were computed for impossible objects with one, two, and three structural violations and were submitted to a repeated measures ANOVA. The hypothesis of an inverse linear relationship between number of structural violations and magnitude of negative priming was again tested with a first-order polynomial contrast. Although neither the effect of number of structural violations $(p=.8)$ nor the polynomial contrast ( $p=.3$, one-tailed) reached significance, the data are clearly consistent with the predicted pattern (see Figure 6). Planned comparison showed that significant negative priming occurred for objects with one structural violation $[t(23)=2.26, p=.034$, one-tailed $]$ but did not reach significance for the remaining objects ( $p \mathrm{~s}>.2$, one-tailed).

The subjects' bias and sensitivity scores for new and old stimuli can be found in Figure 7. Consistent with the results from Experiment 1, subjects' sensitivity increased for old relative to new items $[t(23)=2.61, p=.016]$, and their bias to respond "possible" was greater for old than for new objects $[t(23)=3.48, p=.002]$. An analysis across experiments showed that there were no effects of experiment on bias and sensitivity for new objects and old objects (all $p \mathrm{~s}>.2$ ).

\section{Discussion}

The results from Experiment 2 showed that in the absence of matched objects in the object decision test phase, the pattern of results observed for new and old objects was the same as that observed in Experiment 1 when the matched objects were present. Subjects showed priming for old possible objects and negative priming for old impossible objects. In addition, the negative priming effect for impossible objects appeared to depend on the number of structural violations in the object. Negative priming for old impossible objects reached significance for those objects with one structural violation, but not for those with two or three violations. Also consistent with the results from Experiment 1, subjects' bias to respond "possible" was greater for old than for new items, as was the subjects' ability to discriminate between possible and impossible objects, as measured by sensitivity.

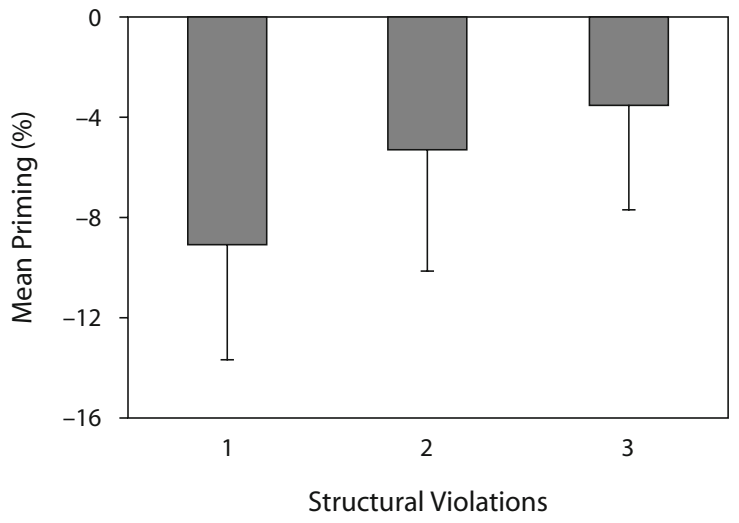

Figure 6. Results from Experiment 2: Mean priming scores for old impossible objects, as a function of the number of structural violations in the object. Error bars represent the standard errors of the means.

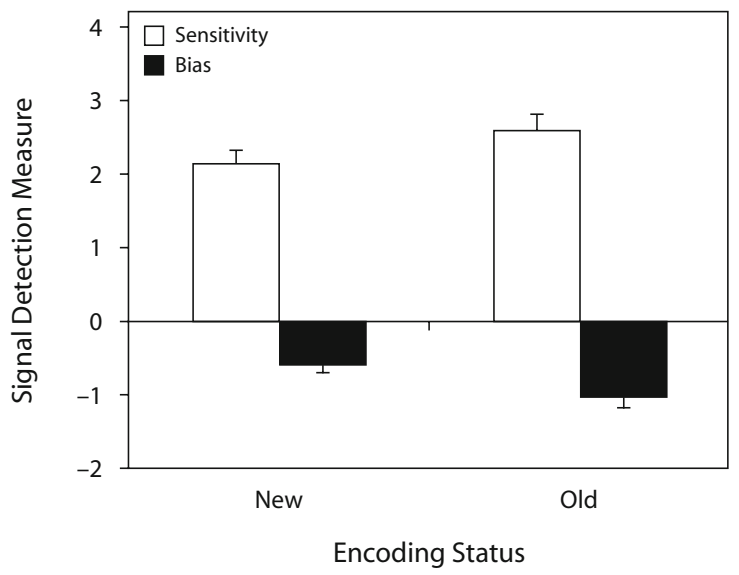

Figure 7. Results from Experiment 2: Mean bias and sensitivity scores for new and old objects. Error bars represent the standard errors of the means. 
The finding of reliable negative priming of old impossible objects in Experiment 2 suggests that negative priming is related to stimulus-specific properties of the impossible objects. Thus, Experiment 2 used the standard implementation of the object decision test as well as the same type of possible objects as in Schacter and Cooper. The only difference from prior studies that did not find negative priming of impossible objects appears to have been the impossible stimuli. In particular, in order to create impossible objects that are closely matched to a possible object, the impossible objects must contain relatively few and subtle structural violations. It is this property that seems to induce reliable negative priming, as indicated by the inverse linear relationship between the number of structural violations and negative priming. Furthermore, the negative priming effect for impossible objects observed in previous studies using the matched-objects design (Ratcliff \& McKoon, 1995; Soldan et al., 2006; Williams \& Tarr, 1997) is also likely to stem from the relatively high structural coherence of the impossible objects used in these studies, not from the presence of the matched objects. The original studies by Schacter and Cooper, by comparison, did not find reliable negative priming for impossible objects, probably because these objects contained too many or too obvious structural violations. These findings underscore the importance of carefully controlling stimulus-specific properties in the study of visual memory for objects.

Taken together, the findings from Experiments 1 and 2 suggest that when clearly differentiable possible and impossible objects are used, the matched objects do not influence object decision performance. The relatively ambiguous nature of the stimuli used by Ratcliff and McKoon (1995), by comparison, may elicit somewhat different processing, which might explain why Ratcliff and McKoon observed an effect of the matched objects on performance for old items (i.e., negative priming for old impossible objects only when the matched objects were presented during the test phase). Procedural differences were also present between studies, but these appear less likely to account for the discrepancy in the results. First, the exposure duration of the test stimuli ranged from 150 to $250 \mathrm{msec}$ in the study by Ratcliff and McKoon, as compared with $33 \mathrm{msec}$ in the present study. However, as described in the Method section, exposure time affects overall object decision accuracy, but it does not appear to affect priming (Schacter \& Cooper, 1993). In addition, the exposure time used in this study was intermediate in comparison with that used by Ratcliff and McKoon and that used by Williams and Tarr (1997; 48 msec masked), yet the pattern of priming reported by Williams and Tarr was quite similar to that reported by Ratcliff and McKoon. Second, the number of stimuli differed across studies, but it is unclear how this could explain differences in priming. Furthermore, none of the three models of object decision priming makes any predictions regarding the effect of exposure duration or number of stimuli on task performance, so it is unclear how such effects, if present, would be interpreted within the context of the three models. (See the online supplement of this article for a list of procedural differences between these and other studies of block-based possible/impossible object decision priming following structural encoding.)

\section{GENERAL DISCUSSION}

There are four important findings from the experiments reported here. First, inclusion of the matched objects in the object decision test phase did not affect task performance - and, by implication, retrieval from explicit memory - when clearly differentiable possible and impossible objects were used. This means that bias effects with a matched-objects design do not provide valid evidence that priming in the standard possible/impossible object decision test is contaminated by explicit memory, unless other, more direct evidence for such contamination is provided. Rather, negative priming effects for impossible objects seem to emerge when these objects have few and subtle structural violations. Second, there was no effect of encoding-to-test delay on priming. Because explicit memory declines over the same time period, this provides further evidence against the view that explicit memory contributes to priming in this task. Third, reliable negative priming for impossible objects can be obtained using standard procedures (Experiment 2), provided that the impossible objects have few structural violations. This indicates that, contrary to Schacter and Cooper's structural-description system model, priming in the possible/impossible object decision test is not supported exclusively by global structural descriptions. Fourth, the finding of priming for old possible objects but not for matched possible objects in Experiment 1 indicates that priming for the type of possible objects used in this study was not based solely on facilitated processing of object parts, but seemed to require also that the object's global 3-D structure be encoded. Taken together, these findings suggest that both local and global 3-D structural descriptions exist and can contribute to priming in the possible/ impossible object decision test. Any successful model of possible/impossible object decision priming, therefore, should refer to both types of representations.

It is unclear why Ratcliff and McKoon (1995) and Williams and Tarr (1997), but not this study, found positive priming for matched possible objects. A possible reason could be related to the structural properties of the possible objects used in each study. Thus, the relatively ambiguous nature of the stimuli used by Ratcliff and McKoon suggests that the global 3-D structure of many of their possible objects was not clearly discernable. This means that subjects probably based their object decisions for both possible and impossible objects mostly on local structural information. Consequently, greater facility in processing previously encountered object parts affected object decision performance for both possible and impossible objects, leading to the priming of old and matched possible and impossible objects.

Williams and Tarr (1997) used possible objects that were clearly identifiable as possible (when given unlimited time), but they found priming for matched possible objects nonetheless. There were procedural differences between this and Williams and Tarr's study (e.g., test phase stimulus 
exposure duration, number of stimuli), but it is unclear how these would account for the discrepancy across studies. A more plausible explanation might be related to differences in stimulus sets. One possibility is that the possible objects used by Williams and Tarr shared structural properties among each other, so that the priming effect for matched possible figures did not reflect facilitated processing of previously encountered parts (from the matching impossible version), but did reflect facilitated processing of the global structure of structurally similar possible objects. The objects in the present study are relatively unique and show little structural similarity to each other. Another possibility is that the global 3-D structure of Williams and Tarr's possible objects was simply more difficult to perceive or encode than the global structure of our possible objects (when viewing was limited), leading the subjects to rely more on parts-based processing. Such differences in the ability to extract the global 3-D structure of objects could potentially arise if the stimuli vary in structural complexity or in orientation (i.e., viewpoint). Even the manner in which structural violations are created and their location (periphery vs. center) might affect stimulus processing and priming of the corresponding possible objects.

Although the present study cannot adjudicate between these alternatives, we tentatively propose the following as an account for the pattern of priming observed in this and prior studies using the possible/impossible object decision test. When the global structural description from a briefly presented possible test figure can easily be extracted, the structural information gathered from purely local features provides little additional evidence that the object is globally coherent. Consequently, priming of possible objects occurs only if global structural processing is facilitated (as in the case of old possible objects), not if processing of only parts of the object is facilitated (as in the case of matched possible objects). By comparison, when the global 3-D structure of a briefly presented possible object is less readily perceived - as with Ratcliff and McKoon's (1995) and, perhaps, Williams and Tarr's (1997) stimulisubjects may rely more on parts-based processing, so that local structural information is more likely to influence object decisions for possible objects, leading to priming of both old possible and matched possible objects. Negative priming of impossible objects always reflects facilitated processing of parts-based structural information and is inversely related to the number of structural violations. This account is speculative, however, and requires further experimentation. We do not discount the observations made by Ratcliff and McKoon and by Williams and Tarr. Rather, we feel that the present results illustrate that a more complete investigation of the effects of stimulus characteristics and testing conditions will reveal a more complex memory phenomenon.

Further support for the view that both local and global 3-D structural descriptions are processed and stored in the brain comes from neuroimaging (Habeck, Hilton, Zarahn, Brown, \& Stern, 2006; Schacter et al., 1995; Soldan, Gazes, Hilton, \& Stern, 2008) and electrophysiological (Friedman \& Cycowicz, 2006; Penney et al., 2000; Soldan et al., 2006) studies, which tend to support the presence of a qualitative difference in the way possible and impossible objects are processed. Whereas the repetition of structurally possible objects routinely elicits neural repetition effects, the repetition of impossible objects produces no measurable neural repetition effects when more than six items intervene between repetitions (Friedman \& Cycowicz, 2006; Penney et al., 2000; Schacter et al., 1995; Soldan et al., 2006). When relatively few (two to six) items intervene between repetitions, possible and impossible objects appear to be associated with different neural repetition effects, as indicated by the anatomical locus and direction (increase vs. decrease) of the effects (Habeck et al., 2006; Soldan, Gazes, et al., 2008).

\section{Implications for Theories of Object Representation}

The parts-based structural descriptions that seem to support negative priming for impossible objects might be based on the kind of volumetric primitives envisioned by Biederman (1987) to underlie object recognition (Hummel \& Biederman, 1992; Hummel \& Stankiewicz, 1996; see also Marr \& Nishihara, 1978). Such representations would consist of only those portions of an impossible object with clearly identifiable 3 -D parts. The relationships between these parts, however, could not be (fully) specified, because no singular globally consistent interpretation of part relations can be generated for impossible objects. Support for the separation of structural descriptions of the parts of an object and descriptions of the spatial relationship between these parts comes from a case study of a patient with a severe deficit in the latter, but not the former, type of process (Behrmann, Peterson, Moscovitch, \& Suzuki, 2006; see also Arguin \& Saumier, 2004; Humphrey, Goodale, Jakobson, \& Servos, 1994). The global structural descriptions that appear to contribute to priming of possible objects, at least under some conditions, might consist of this spatial information about the relative configuration of object components, as well as information about the objects' axes and lines of symmetry.

Another possibility, suggested by Williams and Tarr (1997), is that the structural descriptions of impossible objects are similar to the $2 \frac{1}{2} 2$-D sketch posited by Marr (1982) to precede formation of a global, 3-D, viewpointinvariant representation during vision. Such a $2 \frac{1}{2}-\mathrm{D}$ sketch is thought to specify the surface discontinuities of an object, as well as slant and depth of each surface, relative to the viewer. As such, a $2 \frac{1}{2}-\mathrm{D}$ sketch could be sufficient for representing the global impossibility of a 3-D structure. For possible objects, though, priming was unlikely to be based on a $2 \frac{1}{2}-\mathrm{D}$ sketch in this study, because one would then have expected to find priming for matched possible objects, for which a similar 21/2-D sketch was available from the encoded impossible version of the object. Viewer-based 2-D or 2 $1 / 2-D$ image information and 3 -D structural information probably operate in parallel in the task (Foster \& Gilson, 2002; Hayward, 2003; see also Edelman \& Intrator, 2000; Hummel \& Stankiewicz, 1996). The particular visual representations that are accessed in a particular situation appear to be dictated, at least partially, by the specific stimulus properties and re- 
quirements of the task (Glushko \& Cooper, 1978; Liu \& Cooper, 2001). Carefully controlling for these conditions may lead to a broader understanding of object perception and representation.

\section{The Role of Explicit Memory in Possible/ Impossible Object Decision Test Performance}

Because the influence of explicit memory on priming was not measured directly in this study - nor has it been measured directly in any prior study using the matchedobjects design - the present study provides only indirect evidence regarding its role in the possible/impossible object decision test. This evidence should, therefore, be interpreted cautiously. The finding that delay did not affect priming argues against the influence of explicit memory on task performance and is consistent with findings from prior studies showing that encoding-to-test delays can produce dissociations between performance on implicit and on explicit tests of memory (Goshen-Gottstein \& Kempinsky, 2001; Mitchell \& Brown, 1988; Wiggs, Weisberg, \& Martin, 2006). These data replicate the findings by Soldan et al. (in press), who reported no change in possible/impossible object decision priming in young adults over 1 week with nonmatching possible and impossible stimuli. Finally, the lack of an effect of delay on priming is consistent with findings from many prior studies demonstrating dissociations between priming on the possible/impossible object decision test and performance on parallel explicit recognition tests for the same objects (e.g., Cooper et al., 1992; Schacter \& Cooper, 1993; Schacter et al., 1990; Schacter, Cooper, Delaney, et al., 1991; Soldan, Mangels, \& Cooper, 2008).

One reason Ratcliff and McKoon (1995) were able to provide evidence for the influence of explicit memory on priming in the possible/impossible task-whereas other studies generally failed to find such evidence — could be related to the manner in which explicit memory was manipulated. Studies failing to find evidence for the influence of explicit memory used manipulations that left the object decision test phase unchanged. Ratcliff and McKoon, by comparison, exclusively used test-phase manipulations, such as the inclusion of the matched objects and the imposition of response deadlines and memory loads. They reasoned that requiring subjects to respond quickly or to hold unrelated items in working memory during performance of the object decision test would prevent them from engaging in explicit memory retrieval.

Such retrieval manipulations, however, can be problematic for two reasons. First, they assume that the retrieval manipulation only affects explicit retrieval processes without altering other task-related processes or even implicit memory retrieval. To our knowledge, prior studies have not examined whether response deadlines, memory loads, or highly similar stimuli do, in fact, have dissociable effects on retrieval from implicit and explicit memory during indirect memory tasks. Second, it is unclear how one would evaluate the effectiveness of such retrieval manipulations in actually reducing explicit memory. The reason is that such manipulations are thought to change how subjects utilize or access explicit memory in the process of classifying an object as possible or impossible; they are not thought to change the level of explicit memory for previously seen items themselves (Ratcliff \& McKoon, 1995). ${ }^{2}$ This means that one cannot use subjects' performance on an independent test of explicit memory as a measure of their tendency to utilize or access this memory during the object decision test. Therefore, aside from demonstrating that a retrieval manipulation changes priming, it is unclear how one would verify whether the manipulation had an effect on explicit retrieval. In light of these issues and the available evidence from studies using other manipulations, it appears that priming effects in the possible/impossible object decision test are largely independent of subjects' explicit memory for the figures, but that they do reflect implicit perceptual processes. The possible/impossible object decision task has been, and promises to remain, a fertile paradigm for investigating the structural representation of visual objects.

\section{AUTHOR NOTE}

This work was supported by NIA Grant RO1-AG16714 to Y.S. We thank Brian C. Rakitin for useful comments on an earlier version of the manuscript and Tomislav Pavličić, Iris Warren, and Elaine Gazes for research assistance and data collection. Correspondence concerning this article should be addressed to Y. Stern or A. Soldan, Taub Institute, Columbia University Medical Center, 630 West 168th Street, P \& S Box 16, New York, NY 10032 (e-mail: ys11@columbia.edu, as1578@ columbia.edu).

\section{REFERENCES}

Arguin, M., \& SAUMIER, D. (2004). Independent processing of parts and of their spatial organization in complex visual objects. Psychological Science, 15, 629-633.

Behrmann, M., Peterson, M. A., Moscovitch, M., \& Suzuki, S. (2006). Independent representation of parts and the relations between them: Evidence from integrative agnosia. Journal of Experimental Psychology: Human Perception \& Performance, 32, 1169-1184.

Bentin, S., \& Golland, Y. (2002). Meaningful processing of meaningless stimuli: The influence of perceptual experience on early visual processing of faces. Cognition, 86, B1-B14.

BIEDERMAN, I. (1987). Recognition-by-components: A theory of human image understanding. Psychological Review, 94, 115-147.

Biederman, I., \& Cooper, E. E. (1991). Priming contour-deleted images: Evidence for intermediate representations in visual object recognition. Cognitive Psychology, 23, 393-419.

Bowers, J. S., \& Marsolek, C. J. (EDS.) (2003). Rethinking implicit memory. Oxford: Oxford University Press.

Burgund, E. D., \& MarsoleK, C. J. (2000). Viewpoint-invariant and viewpoint-dependent object recognition in dissociable neural subsystems. Psychonomic Bulletin \& Review, 7, 480-489.

Carrasco, M., \& Seamon, J. G. (1996). Priming impossible figures in the object decision test: The critical importance of perceived stimulus complexity. Psychonomic Bulletin \& Review, 3, 344-351.

Cooper, L. A. (1990). Mental representation of three-dimensional objects in visual problem solving and recognition. Journal of Experimental Psychology: Learning, Memory, \& Cognition, 16, 1097-1106.

CoOper, L. A., \& ScHACTER, D. L. (1992). Dissociations between structural and episodic representations of visual objects. Current Directions in Psychological Science, 1, 141-146.

Cooper, L. A., Schacter, D. L., Ballesteros, S., \& Moore, C. (1992). Priming and recognition of transformed three-dimensional objects: Effects of size and reflection. Journal of Experimental Psychology: Learning, Memory, \& Cognition, 18, 43-57.

Edelman, S., \& Intrator, N. (2000). (Course coding of shape fragments $)+$ (retinotopy) approximately $=$ representation of structure. Spatial Vision, 13, 255-264. 
Foster, D. H., \& GiLSON, S. J. (2002). Recognizing novel 3-D objects by summing signals from parts and views. Proceedings of the Royal Society B, 269, 1939-1947.

Friedman, D., \& Cycowicz, Y. M. (2006). Repetition priming of possible and impossible objects from ERP and behavioral perspectives. Psychophysiology, 43, 569-578.

GAUTHIER, I., \& TARR, M. J. (1997). Orientation priming of novel shapes in the context of viewpoint-dependent recognition. Perception, 26 51-73.

GlushKo, R. J., \& Cooper, L. A. (1978). Spatial comprehension and comparison processes in verification tasks. Cognitive Psychology, 10, 391-421.

Goshen-Gottstein, Y., \& Kempinsky, H. (2001). Probing memory with conceptual cues at multiple retention intervals: A comparison of forgetting rates on implicit and explicit tests. Psychonomic Bulletin \& Review, 8, 139-146.

Habeck, C., Hilton, H. J., Zarahn, E., Brown, T., \& Stern, Y. (2006). An event-related fMRI study of the neural networks underlying repetition suppression and reaction time priming in implicit visual memory. Brain Research, 1075, 133-141.

HAYWARD, W. G. (2003). After the viewpoint debate: Where next in object recognition? Trends in Cognitive Sciences, 7, 425-427.

Henson, R. N. A. (2003). Neuroimaging studies of priming. Progress in Neurobiology, 70, 53-81.

Hummel, J. E., \& Biederman, I. (1992). Dynamic binding in a neural network for shape recognition. Psychological Review, 99, 480-517.

Hummel, J. E., \& Stankiewicz, B. J. (1996). Categorical relations in shape perception. Spatial Vision, 10, 201-236.

Humphrey, G. K., Goodale, M. A., Jakobson, L. S., \& Servos, P. (1994). The role of surface information in object recognition: Studies of a visual form agnosic and normal subjects. Perception, 23, 1457-1481.

ITIER, R. J., \& TAYLOR, M. J. (2004). Effects of repetition learning on upright, inverted and contrast-reversed face processing using ERPs. NeuroImage, 21, 1518-1532.

LIU, T., \& COOPER, L. A. (2001). The influence of task requirements on priming in object decision and matching. Memory \& Cognition, 29 874-882.

MARR, D. (1982). Vision: A computational investigation into the human representation and processing of visual information. San Francisco: Freeman.

MarR, D., \& NishiHara, H. K. (1978). Representation and recognition of the spatial organization of 3-D shapes. Proceedings of the Royal Society B, 200, 269-294

MarsoleK, C. J., \& Burgund, E. D. (2005). Initial storage of unfamiliar objects: Examining memory stores with signal detection analyses. Acta Psychologica, 119, 81-106.

MCBride, D. M., \& Dosher, B. A. (1997). A comparison of forgetting in an implicit and explicit memory task. Journal of Experimental Psychology: General, 126, 371-392.

Mitchell, D. B., \& Brown, A. S. (1988). Persistent repetition priming in picture naming and its dissociation from recognition memory Journal of Experimental Psychology: Learning, Memory, \& Cognition, 14, 213-222.

Penney, T. B., Mecklinger, A., Hilton, H. J., \& Cooper, L. A. (2000). Priming and recognition of novel 3-D objects: Guidance from eventrelated potentials. Cognitive Science Quarterly, 1, 69-92.

PoldRACK, R. (1996). On testing for stochastic dissociations. Psychonomic Bulletin \& Review, 3, 434-448.

RATCLIFF, R., \& McKoon, G. (1995). Bias in the priming of object decisions. Journal of Experimental Psychology: Learning, Memory, \& Cognition, 21, 754-767.

Schacter, D. L., \& Buckner, R. L. (1998). Priming and the brain. Neuron, 20, 185-195.

SCHACTER, D. L., \& COOPER, L. A. (1993). Implicit and explicit memory for novel visual objects: Structure and function. Journal of Experimental Psychology: Learning, Memory, \& Cognition, 19, 995-1009.

SCHACTER, D. L., \& COOPER, L. A. (1995). Bias in the priming of object decisions: Logic, assumption, and data. Journal of Experimental Psychology: Learning, Memory, \& Cognition, 21, 768-776.

Schacter, D. L., Cooper, L. A., \& Delaney, S. M. (1990). Implicit memory for unfamiliar objects depends on access to structural descriptions. Journal of Experimental Psychology: General, 119, 5-24.
Schacter, D. L., Cooper, L. A., Delaney, S. M., Peterson, M. A., \& THARAN, M. (1991). Implicit memory for possible and impossible objects: Constraints on the construction of structural descriptions. Journal of Experimental Psychology: Learning, Memory, \& Cognition, 17, 3-19.

Schacter, D. L., Cooper, L. A., Tharan, M., \& Rubens, A. B. (1991). Preserved priming of novel objects in patients with memory disorders. Journal of Cognitive Neuroscience, 3, 117-130.

Schacter, D. L., Cooper, L. A., \& Treadwell, J. (1993). Preserved priming of novel objects across size transformation in amnesic patients. Psychological Science, 4, 331-335.

SCHACTER, D. L., COOPER, L. A., \& VAlDISERri, M. (1992). Implicit and explicit memory for novel visual objects in older and younger adults. Psychology \& Aging, 7, 299-308.

Schacter, D. L., Reiman, E., Uecker, A., Polster, M. R., Yun, L. S. \& COOPER, L. A. (1995). Brain regions associated with retrieval of structurally coherent visual information. Nature, 376, 587-590.

SNOdGrass, J. G., \& CoRwIN, J. (1988). Pragmatics of measuring recognition memory: Applications to dementia and amnesia. Journal of Experimental Psychology: General, 117, 34-50.

Soldan, A., Gazes, Y., Hilton, H. J., \& Stern, Y. (2008). Aging does not affect brain patterns of repetition effects associated with perceptual priming of novel objects. Journal of Cognitive Neuroscience, 20, 1762-1776.

Soldan, A., Hilton, H. J., Cooper, L. A., \& Stern, Y. (in press). Priming of familiar and unfamiliar visual objects over delays in young and older adults. Psychology \& Aging.

Soldan, A., Mangels, J. A., \& Cooper, L. A. (2006). Evaluating models of object decision priming: Evidence from event-related potential repetition effects. Journal of Experimental Psychology: Learning, Memory, \& Cognition, 32, 230-248.

Soldan, A., Mangels, J. A., \& COOPER, L. A. (2008). Effects of attention on perceptual priming of novel visual objects. Memory, 16, 873-895.

Stern, Y., Hilton, H. J., Liu, T., Gladstone, S., Giaime, A., \& CooPER, L. A. (2000, April). The effect of study-to-test delay on implicit memory for novel objects in elderly and young volunteers. Poster presented at the annual meeting of the Cognitive Neuroscience Society, San Francisco.

Tulving, E., \& Schacter, D. L. (1990). Priming and human memory systems. Science, 247, 301-306.

Wiggs, C. L., Weisberg, J., \& Martin, A. (2006). Repetition priming across the adult life span-The long and short of it. Aging, Neuropsychology, \& Cognition, 13, 308-325.

Williams, P., \& TARR, M. J. (1997). Structural processing and implicit memory for possible and impossible figures. Journal of Experimental Psychology: Learning, Memory, \& Cognition, 23, 1344-1361.

Wixted, J. T., \& EBBESEN, E. B. (1991). On the form of forgetting. Psychological Science, 2, 409-415.

\section{NOTES}

1. The primary aim of the study by Soldan et al. (in press) was to assess the effect of normal aging on priming of familiar and unfamiliar visual stimuli. Only Experiment 1 of that study is of relevance to the present investigation.

2. This was confirmed in a pilot experiment in our laboratory. Specifically, when given a recognition test immediately after the left/right encoding phase, subjects' accuracy in classifying old objects as old (i.e., the probability of hits) was the same whether the matched objects were present or absent during the test phase. The false alarm rate for matched objects, however, was higher than that for new objects, indicating a greater level of familiarity with the matched items than with the new items.

\section{SUPPLEMENTAL MATERIALS}

A table summarizing studies of the possible/impossible object decision test may be downloaded as supplemental materials for this article from mc.psychonomic-journals.org/content/supplemental.

(Manuscript received July 11, 2008; revision accepted for publication October 20,2008.) 